\title{
Levantamento de potenciais criadouros de Aedes aegypti no Campus do Itaperi da Universidade Estadual do Ceará
}

\author{
[Survey of potential breeding sites of Aedes aegypti on Itaperi Campus at Ceará State University]
}

\section{"Artigo Científico/Scientific Article"}

\section{Tobias Saraiva Cavalcante Neto ${ }^{*}$, Mariana Torres Portugal Ramirez ${ }^{\mathbf{1}}$, Victor Reis Galindo ${ }^{\mathbf{1}}$, Lícia Flávia Silva Herculano², Maria Verônica Morais Campello ${ }^{1}$}

\author{
${ }^{1}$ Universidade Estadual do Ceará (UECE), Fortaleza-CE, Brasil. \\ ${ }^{2}$ Universidade Federal de Santa Maria (UFSM), Santa Maria-RS, Brasil. \\ *Autor para correspondência/Corresponding author: E-mail: tobiasscneto@ hotmail.com
}

\begin{abstract}
Resumo
No Campus Itaperi, UECE há fluxo intenso de transeuntes, condições favoráveis ao desenvolvimento e manutenção do Aedes aegypti, grande quantidade de inservíveis, bem como lixo eliminado em áreas extensas. Por esse motivo, objetivou-se fazer um levantamento dos seus potenciais criadouros de Aedes aegypti encontrados no local e sugerir estratégias de controle, com o intuito de reduzir a incidência de novos casos de dengue, zika vírus e chikungunya. Os dados foram obtidos junto ao Distrito técnico de endemias da Regional IV, do município de Fortaleza-CE. Foi realizada também, uma inspeção rigorosa no Campus, com o intuito de observar a situação dos possíveis criadouros e a presença de outros geradores de focos. Foram verificados alguns reservatórios e caixas d'água sem vedação; lajes e caixas dos climatizadores dos blocos contendo água e folhas; poços abertos; escombros derivados de uma reforma, onde foram encontradas larvas de Ae. Aegypti; grande quantidade de inservíveis utilizados pelos comensais ou adaptados como bebedouros e comedouros, para cães e gatos, que vivem à solta no Campus. Diante desse cenário foram sugeridas medidas corretivas, com o intuito de reduzir a população do vetor. Nesse contexto, o Campus necessita de políticas públicas próprias para gerenciar a demanda dos resíduos sólidos, além de mobilizações educacionais, monitoramento periódico do lixo e reforço às ações das brigadas.
\end{abstract}

Palavras-chave: inseto; universidade; vetor.

\begin{abstract}
There is an intense flow of people on Itaperi campus at Ceará State University, favoring conditions for Aedes aegypti development and maintenance, and trash scattered in extensive areas. Thus, this study aims to investigate Aedes aegypti potential breeding sites on campus and suggest strategies of control, in order to reduce the incidence of new cases of dengue, zika, and chikungunya fever. The Technical District of Endemic Diseases (Distrito Técnico de Endemias) from Regional IV in Fortaleza-CE provided the data for this study. A strict inspection was also conducted in the campus in order to observe the situation of possible breeding sites and the presence of other foci. Finding were: some water tanks unsealed; slabs and boxes of air conditioners containing water and leaves; building reform debris; where Aedes aegypti larvae were found; large amount of plastic cups used by diners or turned into bowls for errant dogs and cats from campus. Given this scenario, remedial measures were suggested in order to reduce the vector population. Therefore, the campus needs its own public policies to manage appropriate destination of solid waste, as well as educational mobilizations and periodic garbage inspection.
\end{abstract}

Keywords: insect; university; vector. 


\section{Introdução}

Aedes aegypti (Diptera: Culicidae) é o principal vetor do vírus da dengue, tendo também importância na transmissão de outras arboviroses que acometem o homem como, por exemplo, o Zica vírus e febre Chikungunya (Oliveira et al., 2015). Adaptado ao ambiente urbano, esse mosquito vive em contato com cerca de 2,5 bilhões de pessoas (WHO, 2012), o que os expõem ao risco da transmissão de arboviroses (Sousa et al., 2010).

Estudos demonstram, para todos os países nos quais circula o vírus da dengue, a dificuldade em controlar o Ae. aegypti, devido ao processo de urbanização desordenado, produzindo regiões com alta densidade demográfica, graves deficiências no abastecimento de água e na limpeza urbana e intenso trânsito de pessoas entre as áreas urbanas (Teixeira et al., 2009).

Os ovos de Ae. aegypti são muito resistentes à desidratação. Este aspecto assegura viabilidade dos ovos por meses, em ambientes com baixas umidades (BRASIL, 2008) e favorece a dispersão passiva dos mosquitos. As fêmeas tem preferência por criadouros com água relativamente pobre em matéria orgânica e utilizam para desova uma grande variedade de recipientes comuns do ambiente urbano, como por exemplo, caixas d'água, tonéis, latões, cisternas, frascos e latas vazias, pneus, pratos de vasos, bromélias etc. (Lourenço-de-Oliveira et al., 2008), mas também pode se adaptar às novas circunstâncias geradas pelo homem (Varejão et al., 2005), em esgoto por exemplo (Beserra et al., 2009). Os macrocriadouros, como os tonéis e caixas-d'água, assumem importância maior para a manutenção das altas densidades do vetor da doença. Esses locais resultam da falta de estrutura dos imóveis e do armazenamento inadequado da água, pois, em virtude de irregularidades no abastecimento, a população acondiciona água em recipientes não apropriados, criando condições de receptividade para a proliferação do vetor (Lagrotta et al., 2008).

A compreensão da bioecologia das populações de Ae. aegypti é imprescindível para a elaboração e estratégias de controle.

$\mathrm{O}$ controle vetorial do Ae. aegypti é considerado um dos maiores desafios mundiais e, devido ao seu comportamento, vem demostrando resistência ao controle químico (Tauil, 2006). Os programas de controle de doenças transmitidas por vetores, são orientados a adotarem inseticidas químicos como a principal medida de controle vetorial (WHO, 2012), no entanto estas estratégias são inadequadas e pouco efetivas para o controle da doença (Santos et al, 2014).

Assim, como alternativa, tem-se o controle biológico com peixes larvófagos, porém só funciona em locais onde os criadouros principais de Ae. aegypti são tanques com grandes volumes de água (Tana et al., 2012). Segundo Lefrève (2007) há ainda a necessidade de reformulação das ações educativas, em face da distância entre o conhecimento e mudança de comportamento por parte da população, discussão das estratégias de prevenção e controle mais adequadas

Devido à possibilidade de veiculação das arboviroses na comunidade do Campus do Itaperi, o estudo teve como objetivo fazer um levantamento dos potenciais criadouros de Ae. aegypti e designar estratégias corretivas, com o intuito de reduzir a presença desse vetor.

\section{Material e Métodos}

$\mathrm{O}$ estudo foi realizado entre fevereiro e abril de 2016, na área urbana do Campus do Itaperi da Universidade Estadual do Ceará, como um estudo preliminar para o conhecimento da presença de $A e$. aegypti nessa comunidade. Situada no bairro Itaperi, esta se encontra inserida na Secretaria Executiva Regional IV, da cidade de Fortaleza.

Os dados de análise foram extraídos do Distrito Técnico de Endemias (DTE) da Regional IV, coordenada pelo Núcleo de Controle de Endemias (NUCEN) e Célula de Vigilância Ambiental e Riscos Biológicos (CEVAB), subordinados a Secretária de Saúde do Município de Fortaleza (SMS).

Em paralelo, uma inspeção rigorosa foi feita semanalmente no Campus (Figura 1) com o apoio dos Agentes Comunitários de Endemias (ACEs), com o intuito de observar a presença de potenciais criadouros, tais como depósitos inservíveis, tanques, caixas d'água, cacimbas, cisternas, entulho de construção.

A observação de focos foi realizada pelo ACE com ajuda de escada e de lanterna, sempre que necessário. Paralelamente, utilizou-se uma prancheta na qual apresentava um check list de todos os possíveis macro e microcriadouros do Ae. aegypti, a fim de que tudo fosse vistoriado e anotado.

As larvas coletadas foram identificadas, com o auxílio de chaves dicotômicas de Lane e Whitman (1951), Lane (1953) e Cotrim e Galati (1977), e as descrições das espécies de acordo com Theobald (1903), Borroul (1904), Dyar e Knab 
(1906), Root (1927), Kumm (1933), Pessoa e Galvão (1936), Rozeboom e Komp (1950), Lane e Whitman (1951), Lane (1953), De Oliveira Coutinho e Forattini (1962), Belkin et al., (1971) e Cotrim e Galati (1977). Os criadouros em potencial não foram quantificados, em virtude da sua grande quantidade de dispersão, haja visto que a área trabalhada corresponde um terço da área total do Campus.

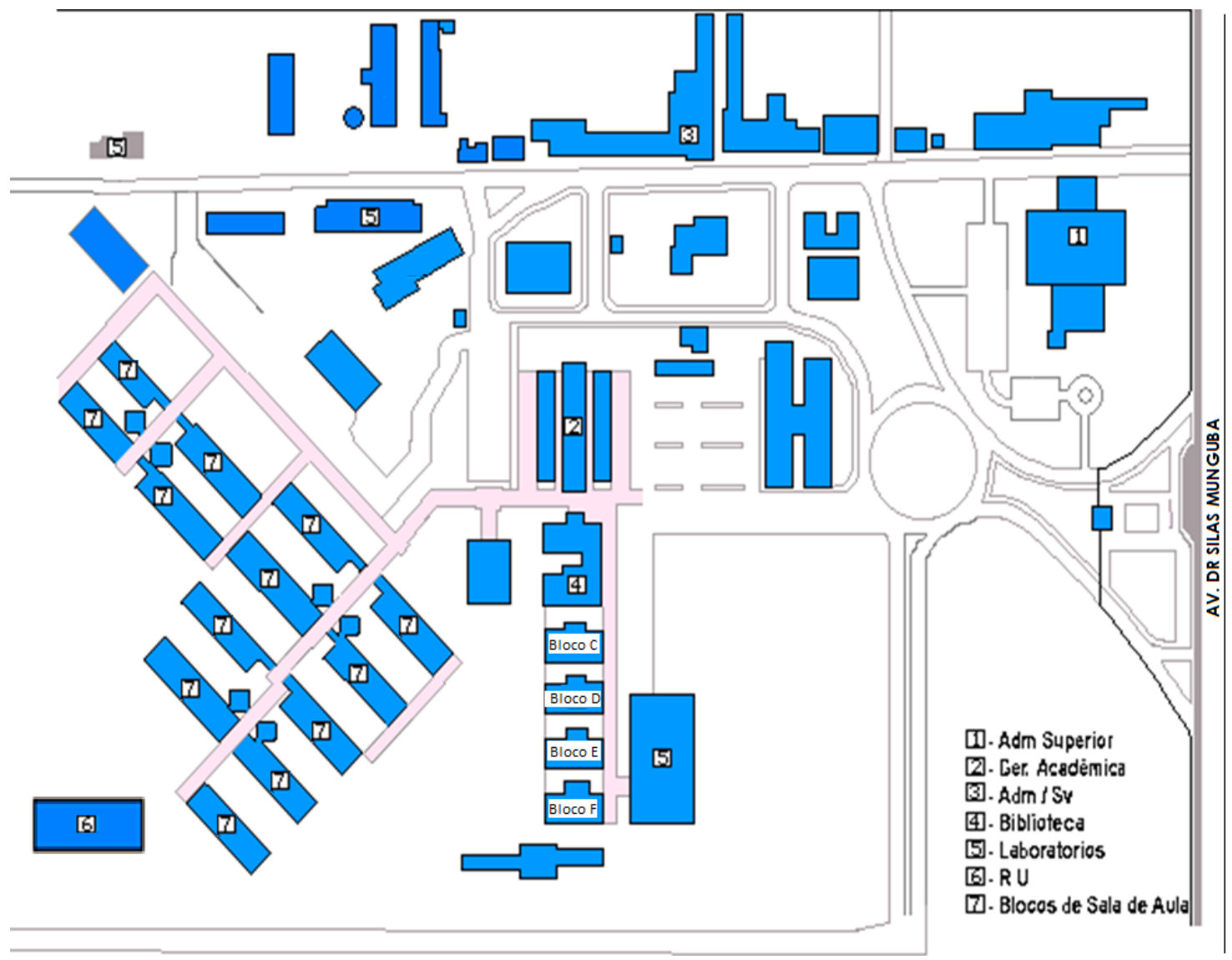

Figura 1. Planta do Campus do Itaperi, demonstrando a localização dos pontos avaliados.

\section{Resultados}

A quantidade de grandes e médios reservatórios se encontram na Tabela 1 . Já na Tabela 2 encontra-se a descrição dos pequenos reservatórios, os locais e sugestões de medidas corretivas. Para os vários tipos de criadouros foram indicadas medidas compatíveis com o diagnóstico. A maioria destes necessitou de substituição, vedação ou reparo, limpeza, correção do declive ou retirada imediata pela Prefeitura do Campus Universitário.

Tabela 1. Potenciais criadouros de grande e médio porte encontrados no Campus do Itaperi, entre fevereiro e abril de 2016.

\begin{tabular}{|c|c|c|c|c|}
\hline \multirow[b]{2}{*}{$\begin{array}{l}\text { Potenciais } \\
\text { criadouros }\end{array}$} & \multicolumn{4}{|c|}{ Condições } \\
\hline & Vedado(a) & $\begin{array}{c}\text { Com } \\
\text { peixes }\end{array}$ & $\begin{array}{c}\text { Sem } \\
\text { vedação }\end{array}$ & Total \\
\hline Tanque & - & 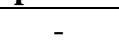 & 7 & 7 \\
\hline Cisterna & 13 & 1 & - & 14 \\
\hline Cacimba & - & - & 2 & 2 \\
\hline Caixa d'água & 7 & - & 13 & 20 \\
\hline Total & 20 & 1 & 22 & 43 \\
\hline
\end{tabular}


Tabela 2. Potenciais criadouros de pequeno porte encontrados no Campus do Itaperi, no período de 19/02/2016 a $24 / 04 / 2016$.

\begin{tabular}{|c|c|c|c|}
\hline Potenciais criadouros & Diagnóstico & Setores & Sugestões \\
\hline Calha dos climatizadores & $\begin{array}{c}\text { Acúmulo de sujidade } \\
\text { e água }\end{array}$ & $\begin{array}{l}\text { Administração } \\
\text { Bloco D }\end{array}$ & $\begin{array}{l}\text { Esvaziamento, limpeza, } \\
\text { correção do declive } \\
\text { ou substituição }\end{array}$ \\
\hline Caixa d'agua de amianto & $\begin{array}{l}\text { Sem vedação, acúmulo } \\
\text { de folhas e água }\end{array}$ & $\begin{array}{l}\text { Biblioteca } \\
\text { Bloco C e D } \\
\text { Bloco E e F }\end{array}$ & $\begin{array}{c}\text { Substituição ou vedação } \\
\text { e limpeza }\end{array}$ \\
\hline Laje & Acúmulo de água & $\begin{array}{l}\text { Biblioteca } \\
\text { Bloco C e F }\end{array}$ & $\begin{array}{c}\text { Desobstrução da laje, } \\
\text { limpeza e correção do declive }\end{array}$ \\
\hline $\begin{array}{l}\text { Depósitos inservíveis } \\
\text { (copos, marmitas vazias, } \\
\text { garrafas plásticas) }\end{array}$ & Acúmulo de água & $\begin{array}{c}\text { Bloco C } \\
\text { Restaurante } \\
\text { Universitário }\end{array}$ & $\begin{array}{l}\text { Esvaziamento e manejo } \\
\text { adequado dos depósitos }\end{array}$ \\
\hline $\begin{array}{l}\text { Receptáculo de água } \\
\text { dos climatizadores }\end{array}$ & Acúmulo de água & Bloco D & $\begin{array}{l}\text { Esvaziamento e manejo } \\
\text { adequado dos depósitos }\end{array}$ \\
\hline Entulho de construção & Acúmulo de água & $\begin{array}{c}\text { Entre Bloco C e D } \\
\text { Atrás do Auditório } \\
\text { Central }\end{array}$ & Retirada imediata do lixo \\
\hline
\end{tabular}

Dentre os potenciais criadouros, as caixas d'água detiveram o maior número, a maioria delas era de amianto, necessitavam de limpeza e estavam sem vedação e/ou sem tampa. Quando vedadas isso era feito com a própria tampa, não havendo proteção da água, contra a postura de qualquer inseto cujo ciclo possa ser desenvolvido na água.

Soma-se a isso, troncos de árvores com fendas, esgotos abertos, caixas de ar condicionado sem declive ou "ladrão", pedúnculos de plantas, além da superfície côncava das lajes dos antigos blocos que comportam a Biblioteca Central, dos blocos C, D, E e F.

Os tanques estavam abertos sem qualquer vedação e sem histórico de limpeza. Foi encontrado foco de Ae. aegypti, na cisterna que se encontra no bloco $\mathrm{S}$, como resultado da manipulação humana e do não vedamento desta.

No Restaurante Universitário (RU) foram encontrados focos nas calhas que recolhem água das chuvas, assim como nas cantoneiras que auxiliam a sustentação do telhado. Entre os blocos $\mathrm{D}$ e E observou-se um acúmulo de inservíveis e recipientes com água para animais irrestritos que se constituem em focos.

Observou-se também inservíveis nas imediações do RU e de outras instâncias acadêmicas e administrativas, tais como: copos e garrafas plásticas, marmitas de alumínio e pequenos recipientes plásticos. Após serem colocados em baldes telados, contendo água, mostraram que houve eclosão de ovos, uma vez que surgiram larvas, que foram identificadas à luz da microscopia como sendo de Culex spp. e Ae. aegypti.

\section{Discussão}

Infestações por Ae. aegypti despertam preocupação visto que constituem um dos fatores relacionados às epidemias de dengue. A presença do vetor, associada a condições ambientais favoráveis, determina a sazonalidade da doença em muitas localidades (Lima et al., 2008), causando grande inquietação as pessoas que transitam pelo campus do Itaperi, seja para estudo, trabalho ou outras atividades diversas.

No Campus foi encontrado calhas entupidas, garrafas, lixo a céu aberto além de caixas d'água, cacimbas e cisternas abertas. Estes resultados corroboram com o estudo de Lagrotta et al. (2008) e Souza-Santos (1999), os quais citam que caixas d'agua, cacimbas e vasos de plantas são grandes mantenedores do vetor por longos períodos.

Foi verificado a grande quantidade de depósitos inservíveis tais como, copos descartáveis, marmitas vazias em diversas áreas do Campus destinados aos animais que ali habitam ou objetos largados que não foram destinados ao lixo, aumentando a possibilidade de manutenção do inseto vetor e ratificando o estudo de David et al. (2009) os quais em condições de campo na cidade do Rio de Janeiro, demonstraram que este tipo de recipiente pode abrigar aproximadamente $20 \%$ dos imaturos de Ae. aegypti encontrados no ambiente.

Com relação as calhas que auxiliam na sustentação do telhado, a laje e os coletores dos climatizadores, estas podem servir como mantenedores do inseto, conforme afirma Miyazaki et al., (2009) em que as calhas de sustentação e dos ar-condicionados podem acumular folhas e dificultar o escoamento de água, 
mantendo populações do mosquito mesmo em períodos com baixas precipitações.

Durante o período do estudo, foram criadas brigadas de combate ao vetor, objetivando sensibilizar a comunidade a reduzir a quantidade de lixo, copos descartáveis e marmitex espalhados pelo Campus e, consequentemente, reduzir as populações do inseto, conforme relata Holcman et al., (2012), em que ações mobilizadoras envolvendo as brigadas, a população e o poder público na forma de mutirões de organização, podem ser uma alternativa, desde que bem planejada e divulgada, com destino adequado do material recolhido.

Ainda que a comunidade seja povoada de indivíduos com maior nível intelectual e dotados de maior consciência política, na totalidade da sua massa, não houve o despertar para uma atitude consciente de mudança do cenário que contribui com a propagação do vetor. Assim, Chiaravalloti Neto et al., (1998), Nicolas et al., (2009) e Freitas et al., (2011) ratificam em seus trabalhos que o homem acumula conhecimentos sobre a ocorrência das doenças do seu meio, assim como suas formas de prevenção e controle, contudo o saber não é usado como instrumento de mudança no cotidiano dos indivíduos.

\section{Conclusão}

O local carece da adoção de políticas públicas relativas ao saneamento, destinação e reaproveitamento de resíduos sólidos e líquidos e de formalizar parcerias com organismos públicos e privados para a realização de mobilizações educacionais e monitoramentos periódicos relacionados à minimização dos agravos à saúde dos seus usuários.

\section{Conflito de interesse} interesse

Os autores declaram não existir conflito de

\section{Agradecimentos}

As pessoas que compõem o Centro de Controle de Zoonoses de Fortaleza, aos agentes de endemias e aos funcionários do Distrito Técnico de Endemias da Secretaria Executiva Regional IV.

\section{Referências}

Almeida, P.S.; Meotti, C.; Almeida, G.S.; Nascimento, J.; Araújo, A.D.; Faccenda, O.; Fernandes, M.G. Infestação de Aedes aegypti (Linnaeus, 1762) (Diptera: Culicidae) determinada por armadilha de oviposição (ovitrampas) no município de costa rica, estado de Mato Grosso do Sul. Revista de Patologia Tropical, 42(3): 331-339, 2013.

Beserra, E.B.; Freitas, E.M.D.; Souza, J.T.D.; Fernandes, C.R.M.; Santos, K.D. Ciclo de vida de Aedes (Stegomyia) aegypti (Diptera, Culicidae) em águas com diferentes características. Iheringia. Série Zoologia, 99: 281-285, 2009.

BRASIL. Ministério da Saúde. Disponível em <http:// www.saude.gov.br> 2008. Acesso em: 23 set. 2017

Chiaravalloti Neto, F.C.; Moraes, M.S.; Fernandes, M.A. Avaliação de resultados de atividades de incentivo à participação da comunidade no controle da dengue em bairro periférico do município de São José do Rio Preto, São Paulo, e da relação entre conhecimentos e práticas desta população, Cadernos de Saúde Pública, 14:101-109, 1998.

David, M. R.; Lourenco-de-Oliveira, R.; De Freitas, R.M. Container productivity, daily survival rates and dispersal of Aedes aegypti mosquitoes in a high income dengue epidemic neighbourhood of Rio de Janeiro: presumed influence of differential urban structure on mosquito biology. Memórias do Instituto Oswaldo Cruz, 104:6, p. 927-932, 2009.

Flauzino, R.F.; Santos, R.F.; Oliveira, R.M. Indicadores socioambientais para vigilância da dengue em nível local. Saúde Social São Paulo, 20(1): 225-240, 2011.

Freitas, R.M.; Rodrigues, C.S.; Almeida, M.C.M. Estratégia intersetorial para o controle da dengue em Belo Horizonte (Minas Gerais), Brasil. Saúde Social São Paulo, 20(3): 773785, 2011.

Holcman, M.M.; Barbosa, G.L.; Andrade, V.R.; Domingos, M.F.; Gomes, A.H.A.; Silva, M.; Wanderley, D.M.V. Infestação por Aedes aegypti em imóveis fechados nas visitas para vigilância e controle vetorial de dengue em municípios do Estado de São Paulo. Boletim Epidemiológico Paulista, 9(104): 14-23, 2012.

Lagrotta, M.T.F.; Silva, W.C.; Souza-Santos, R. Identification of key areas for Aedes aegypti control through geoprocessing in Nova Iguaçu, Rio de Janeiro State, Brazil. Cadernos de Saúde Pública, 24(1): 70-80, 2008. 
Lefrève, A.M.C.; Ribeiro, A.F.; Marques, G.R.A.M.; Serpa， L.L.M.; Lefrève, L. Representações sobre dengue, seu vetor e ações de controle por moradores do município de São Sebastião, Litoral Norte do Estado de São Paulo, Brasil. Cadernos de Saúde Pública 23(7): 1696-1706, 2007.

Lima, E.A.; Firmino, J.L.N.; Gomes Filho, M.F. A relação da previsão da precipitação pluviométrica e casos de dengue nos estados de Alagoas e Paraíba, Nordeste do Brasil. Revista Brasileira de Meteorologia, 23: 2648, 2008.

Lourenco-de-Oliveira, R.; Lima, J.B.; Peres, R.; Alves F.C.; Eiras, A.E.; Codeço, C.T. Comparison of different uses of adult traps and ovitraps for assessing dengue vector infestation in endemic areas. Journal of the American Mosquito Control Association, 24(3): 387-92, 2008.

Miyazaki, R.D.; Ribeiro, A.L.M.; Pignatti, M.G.; Campelo Junior, J.H.; Pignati, M. Monitoramento do mosquito Aedes aegypti (Linnaeus, 1762) (Diptera: Culicidae), por meio de ovitrampas no Campus da Universidade Federal de Mato Grosso, Cuiabá, Estado de Mato Grosso. Revista da Sociedade Brasileira de Medicina Tropical, 42(4): 392-97, 2009.

Nelson, M.J. Aedes aegypti: biología y ecología. Organización Panamericana de la Salud, Washington, 1986, 50pp.

Nicolas, S.; Andrea, R.; Gabriela, C. Información, conocimiento y percepción sobre el riesgo de contraer el dengue en Argentina: dos experiencias de intervención para generar estrategias locales de control. Cadernos de Saúde Pública, 25: 137-148, 2009.

Oliveira, A.S.M.; Sousa, A.L.L.; Silva, R.C.; Carmo Filho, J.R. Estudo ecológico da dengue em Araguaína-TO entre 2001 e 2010. Estudos, 42(4): 517-526, 2015.

Santos, S. L.; Parra-Henao, G; Silva, M.B.; Costa e Silva, A.; Silva, L.G. Dengue in Brazil and Colombia: a study of knowledge, attitudes, and practices. Revista da Sociedade
Brasileira de Medicina Tropical, 47: 783787, 2014.

Souza, S.S.; Silva, I.G.; Silva, H.H.G. Associação entre incidência de dengue, pluviosidade e densidade larvária de Aedes aegypti, no Estado de Goiás. Revista da Sociedade Brasileira de Medicina Tropical, 439(2): 152-155, 2010.

Souza-Santos, R. Fatores associados à ocorrência de formas imaturas de Aedes aegypti na Ilha do Governador, Rio de Janeiro, Brasil. Revista da Sociedade Brasileira de Medicina Tropical, 32(4): 373-382, 1999.

Tana, S.; Abeyewick, W.; Arunachalan, N.; Espino, F.; Kittayapong, P.; Wai, K. T.; Horstick, O.; Sommerfeld, J. Eco-bio-social research on Dengue in Ásia: general principles and a case study. In: Charrón, D. Ecohealth research in practice. Springer: Ottawa. 2012. p.173-184.

Tauil, P.L. Perspectivas de controle de doenças transmitidas por vetores no Brasil. Revista da Sociedade Brasileira de Medicina Tropical, 39(3): 275-277, 2006.

Teixeira, M.G.; Costa, M.C.N.; Barreto, F.; Barreto, M.L. Dengue: vinte e cinco anos da reemergência no Brasil. Cadernos de Saúde Pública, 25: 7-18, 2009.

Tilak, R.; Gupta, M.V.; Suryam, M.V.; Yadav, J.D.; Gupta, B.K.K.D. A laboratory investigation into oviposition responses of Aedes aegypti to some common household substances and water from conspecific larvae. Medicine Journal Arm for India, 61: 227229, 2005.

Varejão, J.B.M.; Santos, C.B.D.; Rezende, H.R.; Bevilacqua, L.C.; Falqueto, A. Criadouros de Aedes (Stegomyia) aegypti (Linnaeus, 1762) em bromélias nativas na cidade de Vitória, ES. Revista da Sociedade Brasileira de Medicina Tropical, 38(3): 238-240, 2005.

World Health Organization. Fact sheets. In: Dengue, march 2012 [cited 30 October 2012]. Disponível em: $<$ http://www.wpro.who.int/mediacentre/facts heets/fs_09032012_Dengue/en/index.html.> Acesso em: 13 out. 2015. 